\title{
以培养自我调节学习能力为导向的分析化学实验混合教学模式
}

刘云凤，阎小青，乔增杰 ${ }^{*}$

山西医科大学公共卫生学院，太原 030001

摘要: 为探索合适的教学模式以培养自我调节学习为基础的终身学习能力, 在分析化学实验的教学中, 将学生按班级分 为实验组和对照组, 实验组采用了以 “设计布鲁姆认知教育目标、融合翻转课堂模式和问题导向教学” 等为主线的混合 教学模式, 对照组依然采用传统教学模式, 最后根据访谈、教学平台留言和统一的测验题等方式评价教学效果。结果表 明混合教学模式的教学效果优于传统教学, 且实验组所有学生都愿意继续接受这种新的教学模式。教学实践证明, 学生 在制定自身学习目标及建立相应的自我调节学习策略方面有明显的改进。

关键词: 自我调节学习; 混合教学模式; 分析化学实验; 翻转课堂

中图分类号: G642; O6

\section{The Mixed Teaching Mode to Cultivate the Ability of Self-Regulated Learning in Analytical Chemistry Laboratory}

\author{
Yunfeng Liu, Xiaoqing Yan, Zengjie Qiao * \\ School of Public Health, Shanxi Medical University, Taiyuan 030001, China.
}

\begin{abstract}
In the analytical chemistry laboratory, students were divided into the experiment group and the control group. We adopted the mixed teaching mode, which highlighted the designing Bloom's cognitive goals for education, as well as blending the flipped classroom and the problem-oriented teaching for the experiment group to explore an appropriate teaching mode, aiming at fostering the ability of lifelong learning mainly based on the self-regulated learning. By contrast, the traditional teaching mode was used in the control group. At the end of the course, the effect of the mixed teaching mode was evaluated according to interviews, messages on the teaching platform and the unified test. The results showed that the teaching effect of the mixed teaching mode was better. Moreover, all the students of the experiment group preferred the mixed teaching mode. The teaching practice proved that it was favorable for students to set study goals and build the corresponding self-regulated learning strategies.
\end{abstract}

Key Words: Self-regulated learning; Mixed teaching mode; Analytical chemistry laboratory;

Flipped classroom

随着信息技术的迅猛发展, 新的知识和技术如雨后春笋, 层出不穷、飞速更新, 随之教学方式 也在不断发生变化, 如翻转课堂、慕课、金课以及终身学习等新型教学方式或概念的兴起, 都体现 了学习早已不再是单一的学校活动和教师授业, 而是学习者自我调节学习的一种能力。

自我调节学习在 1986 年被首次提出, 被定义为动机、认知和行为之间的相互作用 ${ }^{[1]}$ 。其最显著 的特征是学习者对自己的学习有实际的控制, 掌控和引导认知和动机去实现自己的学习目标 ${ }^{[2,3]}$ 。近

收稿: 2020-06-12; 录用: 2020-07-17; 网络发表: 2020-08-17

“通讯作者, Email: zengjieqiao@163.com

基金资助：山西医科大学教育教学改革研究项目(XJ2018062) 
年来, 互联网+为自我调节学习提供了更广阔的平台, 发展基于信息技术为基础的教育教学方式也越 来越重要, 教师也被鼓励充分利用信息技术和高质量网络教育资源从而更新教学观念、改进教学方 式等。

“分析化学” 是卫生检验与检疫、环境科学、化学等专业的专业基础课或专业核心课, 该课程 实践性较强, 对提高学生实验技能、培养学生自我调节学习能力是很重要的。近年来我校分析化学 课程一直开展设计性实验, 结果发现, 在唤起学生学习兴趣和提高自我调节学习能力等方面, 均有 较好的效果, 而在建立学习目标、笁选整理资料以及自主性深度思考、分析、解决问题等构建完整 有效的自我调节学习策略方面依然存在较大的培养空间。

目前, 对于引导学生建立自我调节学习策略的研究较为少见, 而策略是培养自我调节学习能力 中必不可少的环节。为此, 在分析化学实验进一步改革中, 通过制定不同层次的学习目标, 采用翻 转课堂和问题导向教学方法, 线上和线下混合式教学, 以期学生收 “鱼” 的同时重在获 “渔”, 旨 在提升其自我调节学习能力, 重在引导学生在学习中设定自身的学习目标, 并为实现目标而思考、 分析提出一系列层层递进的问题, 制定出与之相关的策略, 从而形成一整套环环相扣、层层递进、 相互补充、相互促进、相得益彰的自我调节学习策略。

\section{1 研究对象与方法}

\section{1 研究对象}

以我校卫生检验与检疫专业的分析化学实验为例, 以三年级学生(共 65 人)为研究对象, 其中 1 班 31 位学生(男生 6 人, 女生 25 人)为实验组, 年龄 $20.16 \pm 0.89$ ) 岁, 前两年学业成绩为(79.43 26.06$)$ 分, 2 班 34 位学生(男生 9 人, 女生 25 人)为对照组, 年龄 $(19.76 \pm 0.78)$ 岁, 前两年学业成绩为(76.98 6.46)分。经统计分析, 两个班的学生在年龄、性别和前两年学业成绩方面均无统计学差异。

\section{2 研究方法}

两个班由同一位教师分班授课, 实验组采用混合教学模式, 对照组采用传统教学模式。

\section{2 分析化学实验混合教学模式的实施}

\section{1 实施方案}

教学大纲中所有验证性实验的教学均以培养学生建立自我调节学习策略为导向, 分确定学生学 习目标、构建翻转课堂模式、设计教学相关问题三个方面进行。具体方案见表 1 。

表 1 分析化学实验混合教学模式实施方案

\begin{tabular}{|c|c|c|c|c|}
\hline 阶段 & 角色 & 确定学生学习目标 & 构建翻转课堂模式 & 设计教学相关问题 \\
\hline \multirow[t]{2}{*}{ 课前 } & 教师 & 设计布鲁姆认知教育六层次教学目标 & $\begin{array}{l}\text { 分模块(名称、目的、原理、用品、内 } \\
\text { 容、注意事项等)录制视频 }\end{array}$ & 制定预习测验单 \\
\hline & 学生 & 制定自身学习目标及相应的学习方式 & 观看视频、完成预习任务 & 完成预习测验单, 提出预习中的问题 \\
\hline \multirow[t]{2}{*}{ 课中 } & 教师 & 针对目标提出系列问题 & $\begin{array}{l}\text { 组织活动(提问实验相关问题, 提出讨 } \\
\text { 论话题, 跟踪实验随时指导) }\end{array}$ & $\begin{array}{l}\text { 提出与实验相关的理论问题和操作问 } \\
\text { 题 }\end{array}$ \\
\hline & 学生 & 通过实验、思考、讨论达成目标 & 参与活动, 随时交流讨论, 操作实验 & $\begin{array}{l}\text { 抢答, 疑难点进行组内、组间讨论, } \\
\text { 敢于质疑、提出学习中的疑问 }\end{array}$ \\
\hline \multirow[t]{2}{*}{ 课后 } & 教师 & 引导学生制定下一步学习目标 & $\begin{array}{l}\text { 反思教学(课前任务、课上活动, 评阅 } \\
\text { 实验报告) }\end{array}$ & 团队讨论教学中存在的问题 \\
\hline & 学生 & 制定下一步学习的目标 & $\begin{array}{l}\text { 交流讨论; 撰写实验报告, 重点写结 } \\
\text { 果和讨论以及学习心得 }\end{array}$ & 讨论疑难点, 提出新的问题 \\
\hline
\end{tabular}




\section{2 实施过程}

\subsection{1 课前重在制定目标、录制视频}

在传统实验课堂中, 通常学生对要达到的学习目标、实验中的问题极少思考。为此, 为引导学 生有目的地开展实验、提升自我调节学习能力, 教学改革中按照布鲁姆认知教育目标的六层次来设 计教学目标并做成相应的测验单。如教学中 “氧化还原滴定法测定葡萄糖注射液中葡萄糖的含量” 预习测验单见表 2 。

\section{表 2 “氧化还原滴定法测定葡萄糖注射液中葡萄糖的含量” 预习测验单}

\begin{tabular}{|c|c|}
\hline 布鲁姆认知教育目标 & 预习测试题 \\
\hline 知道 & 实验中氧化葡萄糖的物质是 $(\quad$ )。A. 碘单质 $\quad$ B. 次磺酸根离子 $\quad$ C. 碘酸根离子 $\quad$ D. 碘负离子 \\
\hline 理解 & 硫代硫酸钠溶液在—溶液中较稳定。A. 弱碱性 B. 强碱性 C. 弱酸性 D. 强酸性 \\
\hline 应用 & 滴定中加入指示剂的时间是 $(\quad)$ 。 A. 滴定开始 B. 滴定过程中 $\quad$ C. 近终点 D. 滴定结束后 \\
\hline 分析 & 硫代硫酸钠溶液滴定析出的碘时滴定速度如何控制, 滴定开始应__ , 近终点应__ (从慢摇快滴、 \\
\hline & 快摇慢滴、快摇快滴、慢摇慢滴中选择)想想为什么? \\
\hline 评估 & 实验中发现只滴了几滴硫代硫酸钠溶液，溶液便褪色，可能是什么原因？ \\
\hline 创造 & 请查阅相关资料或文献。测定葡萄糖注射液中葡萄糖含量还可以采用什么方法？最新版中国药典中采用的 \\
\hline & 方法及其原理？将所查资料按照参考文献格式列出 \\
\hline
\end{tabular}

通过应用混合教学模式, 学生制定学习目标的最大进步是逐步趋向于不再照抄教材中的 “实验 目的” , 而是结合自身的实际学习情况, 制定出更细微、明确且可操作的目标。如实验 “氧化还原 滴定法测定葡萄糖注射液中葡萄糖的含量” 中学生制定的目标与教材中实验目的对比见表 3 。再如 在设计性实验的学习目标中, 学生不再单纯地参照教材中的 “实验目的”, 而是倾向于以提升查阅 整理资料、合理分工合作、主动分析和解决实验中的问题等能力为目标。

此外, 学生通过预习后能提出与理论和操作细节有关的一些问题, 如 “葡萄糖具有还原性, 碘 液具有氧化性, 为什么不通过直接反应来测定葡萄糖含量？” “氢氧化钠溶液采用什么仪器加入? 缓慢滴加氢氧化钠溶液时, 缓慢到什么程度? ”等。

表 3 “氧化还原滴定法测定葡萄糖注射液中葡萄糖的含量”目标对比

\begin{tabular}{ll}
\hline 教材 ${ }^{[4]}$ 中实验目的 & 学生制定目标 \\
\hline 掌握返滴定法测定葡萄糖含量的原理和方法; 进一步 & 巩固所学理论知识; 理解葡萄糖含量的测定原理和结果计算公式; 能够准 \\
熟悉酸式滴定管的操作; 练习有色溶液滴定时体积的 & 确快速配制溶液, 熟悉微量滴定管的操作, 做到快速排气和装液; 学会使 \\
读法和碘量瓶的使用 & 用碘量瓶; 进一步练习查阅、整理并篮选资料; 练习用 $t$ 检验处理数据 \\
\hline
\end{tabular}

短小精悍的在线视频有利于学生集中注意力和随时随地学习, 为此, 教学中将实验名称、实 验目的、实验用品、实验内容、注意事项、仪器操作等几个模块录制成视频片段, 每个视频时长为 0.5-6 min。学生通过课余时间完成视频学习, 从而给课堂上留出更多的实验操作和讨论时间。

\subsection{2 课中重在提出问题、操作实验}

问题是打开思维的大门、走向创新的源泉。然而在以往的教学中, 很少学生能提出问题。为更 好地引导学生独立思考、提出问题并应用于自我调节学习策略的构建中, 课堂教学中所有验证实验 均设计了与理论知识和操作要点有关的问题, 如 “氧化还原滴定法测定葡萄糖注射液中葡萄糖的含 量”课堂问题见表 4。带着问题做实验, 更容易知其然知其所以然。

因课前已提示学生预习的内容和思路, 所以实验课堂上这一系列问题(由教师或学生提出)多数 采用快问快答的方式, 个别不能及时答出的疑难问题边实验边讨论。根据实验情况, 学生的最大进 步在于主动性地通过组内、组间讨论和尝试实验去解决疑难问题, 而不是像以往遇到问题直接求助 于老师。例如实验中的典型问题: 加入氢氧化钠的量和速度的控制, 学生很容易为追求速度而快速 
加入氢氧化钠, 为追求浅黄色的现象而加入过多的氢氧化钠, 最终发现硫代硫酸钠滴加几滴甚至不 加便已达到终点, 计算出的结果与葡萄糖含量的标示值也严重不符。学生边讨论边估算出实验中所 需要加入的氢氧化钠和盐酸的量, 并采用慢速滴加氢氧化钠的方式多次尝试实验。经过他们主动的 讨论、实验成就感也较高, 从而乐于实验中。

表 4 “氧化还原滴定法测定葡萄糖注射液中葡萄糖的含量” 课堂问题

\begin{tabular}{|c|c|}
\hline 理论问题 & 操作问题 \\
\hline 1) 滴加氢氧化钠溶液的目的? & 1) 本实验中的葡萄糖溶液需用多少毫升? 配制多少? \\
\hline $\begin{array}{l}\text { 2) 理论计算需要滴加 } \mathrm{NaOH} \text { 多少毫升? } \mathrm{NaOH} \text { 的量加太多有什 } \\
\text { 么影响? }\end{array}$ & $\begin{array}{l}\text { 2) 本实验中的葡萄糖、碘液、 } \mathrm{NaOH} \text { 溶液、 } \mathrm{HCl} \text { 溶液分别用 } \\
\text { 哪种仪器取用? }\end{array}$ \\
\hline $\begin{array}{l}\text { 3) 放置 10-15 } \mathrm{min} \text { 后, 理论上推测溶液颜色应该是什么? } \\
\text { 4) 加 } 6 \mathrm{~mol} \cdot \mathrm{L}^{-1} \mathrm{HCl} \text { 的目的是什么? }\end{array}$ & $\begin{array}{l}\text { 3) 为什么要慢慢滴加稀 } \mathrm{NaOH} \text {, 若加入 } \mathrm{NaOH} \text { 速度过快, 会 } \\
\text { 产生什么后果? }\end{array}$ \\
\hline 5) 酸化后未及时用 $\mathrm{Na}_{2} \mathrm{~S}_{2} \mathrm{O}_{3}$ 标准溶液滴定, 会有什么影响? & 4) 实验中若出现 “ $\mathrm{Na}_{2} \mathrm{~S}_{2} \mathrm{O}_{3}$ 溶液滴定前就已是终点” 的情 \\
\hline 6) 实验中若出现溶液有淡黄色浑浊的现象并伴有刺激性气味, & 况, 则可能是什么原因? \\
\hline \multirow[t]{2}{*}{ 可能是什么原因引起的? } & 5) 若不慎将碘液弄在手上或在白大社上, 分别如何处理? \\
\hline & 6) 测定时滴定管中装什么溶液? 用什么类型的滴定管? \\
\hline
\end{tabular}

\subsection{3 课后重在反思教学、整改方案}

根据课堂教学效果、实验报告学习心得、e-教学平台反馈信息等, 教学团队进行教学反思和讨 论, 并逐渐修改完善教学模式。学生需要反思自己制定的学习目标是否已达到, 下一步怎么改正。

以往实验中, 学生课后反思几乎全部集中在完成教材中的思考题。经过混合教学模式后, 学生 课后反思最大的变化主要在于深入探讨理论问题(如碘遇淀粉变蓝的机理)、思考实验细节操作(如天 平的选择、每种试剂的取用工具或仪器选择), 尤其是可以估算试剂用量和结合统计学方法处理数据 等。另外能够积极主动查阅资料, 如学生实验报告中的反思给出了处理含重铬酸钾废液的几种方法, 此外没有达成的学习目标通过查阅资料进一步实现或设计到下次课的环节中。

可能由于混合教学模式时间短、次数少, 虽然有多数学生可以制定出适合符合自己的学习目标, 但是目标相对较散乱, 还缺乏一定的系统性, 后期的教学中计划建议学生归类并有层次地列出目标 细节。此外, 实验后的反思写的不是很深刻、全面, 建议学生从理论问题、实际问题、方法拓展等 方面思考总结。

\section{3 效果评价}

根据实验组在网络教学平台的留言, 结合访谈, 定性评价学生对混合教学模式的意愿程度; 实 验组和对照组均采用相同试题进行随堂限时测试, 评价混合教学模式的教学效果。

\section{4 统计分析}

对于实验随堂测试成绩, 采用 SPSS 17.0 软件, 独立样本 $t$ 检验进行统计学分析, $P<0.05$ 认为 有统计学差异。

\section{3 教学效果}

根据实验组的反馈, $100 \%$ 的学生愿意继续采用混合教学模式, 因为这种教学模式使得他们做实 验目的性更强, 能列出明确的学习目标, 有助于理清实验思路与判断实验中可能的问题及应对措施, 与此同时，熟悉了查阅文献资料的方式，相应地也提升了自我调节学习能力。

为评价混合教学模式的教学效果, 设计了一系列题目 (含操作要点和制定下次课的学习目标及相 应的达标措施)并进行随堂限时测试。实验组和对照组测试平均成绩分别为 $65.35 \pm 9.44 、 60.16 \pm 8.57$, 通过独立样本 $t$ 检验分析可知, $t$ 值为 $2.325, P$ 值为 $0.023(<0.05)$, 两组成绩有统计学差异。且根 据各分数段成绩分布情况(见表 5), 实验组成绩明显优于对照组。可见, 实验组取得了较佳的教学效果。 
大学 化 学 Univ. Chem. 2021, 36 (1), 2006046 (5 of 5)

表 5 实验组和对照组测验题成绩各分数段内分布情况

\begin{tabular}{ccc}
\hline 分数区间 & \multicolumn{2}{c}{ 百分比 } \\
\cline { 2 - 3 } & 实验组 & 对照组 \\
\hline$<60$ & $23 \%$ & $38 \%$ \\
{$[60,70)$} & $48 \%$ & $56 \%$ \\
{$[70,80)$} & $19 \%$ & $6 \%$ \\
{$[80,90)$} & $10 \%$ & $0 \%$ \\
\hline
\end{tabular}

\section{4 讨论}

根据普通高等学校本科专业类教学质量国家标准, 卫生检验与检疫专业毕业生应具有终身学习 能力、批判性思维能力、创新能力和一定科研发展潜能。分析化学实验作为卫生检验与检疫专业的 核心基础性实验, 除了可为后续课程的学习奠定扎实的基础知识和熟练的基本技能外, 也会影响到 以后的学习方式。因此, 分析化学实验实施以培养学生自我调节学习能力为导向的教学改革, 对其 他课程的教学改革有一定的辐射效应以及对培养学生终身学习能力等有一定的促进作用。近年来, 不 少教育教学工作者以培养自主学习能力为目的对分析化学实验进行了一系列改革探索 ${ }^{[5-9]}$, 并均取得 了较好的效果, 但也存在一定的挑战或改进空间, 如设计性实验需要师生投入大量的课余时间, 建 立虚拟实验室需要有足够经费的支持等。

与已有方法相比, 本文中的混合教学模式最大的优势在于引导学生设定明确的学习目标, 提出 从知识到技能到创新等各层次的问题, 利于指引学生建立自我调节学习策略。

根据教学中的问题并结合学生的反馈, 混合教学模式依然存在较大的改进提升空间, 例如合理 分配每次教学目标的数量、深度、广度, 旨在提升学生自我调节学习能力的同时又不加重学生课余 负担; 加强视频的艺术性以唤起学生学习兴趣; 呼吁校内网络技术的大力支持, 从而为学习提供更 宽阔、便利的学习平台; 有效整合网络教学资源以达到学习的高效性。

\section{5 结语}

本文中探讨的混合教学模式在 “分析化学实验” 课程中的应用取得了较好的教学效果, 利于培 养学生自我学习调节能力的同时也有助于提升学生的信息素养。教学有法, 但无定法, 新的技术、 新的模式对教师也提出了新的挑战, 需要教师花时间、花精力对教学模式的探究继续精雕细环。

\section{参 考 文 献}

[1] Bandura, A. Social Foundations of Thought and Action: a Social Cognitive Theory; Prentice Hall: Englewood Cliffs, NJ, USA, 1986.

[2] Boekaerts, M.; Cascallar, E. Educ. Psychol. Rev. 2006, 18 (3), 199.

[3] 路海东, 张丽娜. 东北师大学报(哲学社会科学版), 2011, No. 6, 145 .

[4] 张加玲主编. 分析化学实验. 北京: 人民卫生出版社, 2015: 69-70.

[5] 柳玉英, 王平, 王粤博, 刘青, 萄红桃, 张天, 范慧清. 实验室研究与探索, 2018, 37 (4), 217.

[6] 吴艳芳. 教育现代化, 2018, No. 25, 193.

[7] 岑瑶, 胡琴, 魏芳弟, 许贯虹. 基础医学教育, 2018, $20(2), 151$.

[8] 张加玲, 刘云风, 阎小青. 黑龙江教育(高教研究与评估), 2018, No. 6, 42 .

[9] 刘晓庚, 苏畅, 周佳虹, 王芳敏, 邓梦浩, 邰佳, 李心悦, 彭冬梅. 农产品加工, 2019, No. 5, 115. 